Reprinted from: Causality and Locality in Modern Physics \& Astronomy: Open Questions and Possible Solutions. G. Hunter \& S. Jeffers Eds. Dordrecht: Kluwer Academic, 1998.

\title{
THE ORIGIN OF COSMOLOGICAL REDSHIFT IN SPIN EXCHANGE VACUUM COMPACTIFICATION AND NONZERO REST MASS PHOTON ANISOTROPY
}

\author{
Applications of Quantum Gravity Part I \\ RICHARD L. AMOROSO,* MENAS KAFATOS, and PAULE ECIMOVIC \\ 1-Noetic Advanced Studies Institute, \\ 120 Village Square \#49 Orinda, Ca 94563-2502 USA, *contact author \\ 2-Center for Earth Observing \& Space Research, \\ George Mason Univ, Fairfax, Va 22030-4444 USA \\ 3-Department of Philosophy, \\ University of Ljubljana, Askerceva 2, 1000 Ljubljana, Slovenia
}

\begin{abstract}
Einstein's refinement of Newtonian gravitation repeated for General Relativity (GR) by quantum cosmology requires inflationary models of the universe to be critically reevaluated; the Hubble redshift is shown not to result from Doppler velocity, but anisotropic coupling to vacuum zero point fluctuations through harmonic structure described in terms of the Wheeler - Feynman absorber theory of radiation in the context of a Dirac vacuum and compactification dynamics. The quantum gravity of the comoving hyperstructure of a universe topologically both open and closed like a Klein bottle, also implies that frequency shift in photon propagation over cosmological distances is an inherent part of the spin exchange process, removing the ad hoc criticism of the well known Vigier theory of 'tired light'.
\end{abstract}

\section{Introduction}

This paper on photon propagation applies spin exchange quantum gravity (SEQG) to issues of cosmology. SEQG requires photon rest mass anisotrophy and a radical new view of compactification. The selfreferential flavor of general relativity's (GR) equivalence principle induced conformal map between a curved Einstein-Reimannian 4-space and a locally conformally flat Lorentzian spacetime manifold shelved the propagation problem inherent in a 'Maxwellian ether' after the null results of the Michelson -Morley experiment; but Einstein said relativity did not compel us to exclude the possibility of an ether - namely spacetime itself. Since GR endows space with physical qualities; 'space without ether is unthinkable' (Einstein, 1922). Photon anisotropy requires vacuum zero point coupling, and its propagation can no longer be considered independent of the Dirac vacuum (Amoroso, Kafatos \& Ecimovic, 1998b). The fluctuation of the vacuum zero point field is consistent with the Sakharov-Puthoff model of gravitation (Sakharov, 1968; Puthoff, 1989).

Einstein, Schrodinger, and de Broglie have attested to the significance of non zero photon rest mass. Frequency anisotrophy results from a putative $10^{-65} \mathrm{~g}$ periodic nonzero photon rest mass according to $E=h v=M C^{2}\left[1-v^{2} / c^{2}\right]^{-1 / 2}$ (Narlikar, Pecker \& Vigier, 1991). The Wheeler-Feynman absorber theory of radiation as refined by Cramer, 1986 and others is utilized because the emitter-absorber transaction model is logically consistent with SEQG. 


\subsection{SOME PHILOSOPHICAL AND METAPHYSICAL CONSIDERATIONS OF PROBLEMS WITH THE BIG BANG'S THEORETICAL CONSISTENCY}

The Big Bang (BB)is obviated by invalidating Einstein-Friedman universes upon which it is based. The field equations of GR allow for singularities, the existence of which has been used to predict black holes and BB inflationary origins of a temporal universe; but both GR and quantum theory (QT) are known to be incomplete. The BB although highly successful cannot claim logical consistency. This was the state of Newtonian mechanics before it was superseded. It is not possible for an event to initialize inflation from an era of infinite entropy without violating the law of conservation of energy. Therefore scientific justification for a BB era is not possible (Narlikar, 1996). Many other inconsistencies with the BB interpretation, are passionately debated in the literature.

The standard BB model is founded on strong observational data; prompting many to accept it unconditionally. While the empirical data are correct; the interpretation relies on an incorrect metaphysical basis. The crisis facing scientific epistemology has come full circle to a similitude of Galileo's time when sound philosophical deduction failed.

One of us (Kafatos, 1990) has described 3 types of nonlocality. The first two commonly understood as: Type I - spatial nonlocality and: Type II - temporal nonlocality arise from quantum theory and form a complementarity. Type III nonlocality is inaccessible to the techniques of the standard model of quantum theory because of the measurement problem. Type III nonlocality refers to the undivided wholeness of the unified field (Kafatos \& Nadeau, 1990; from which the elemental principles of quantum gravity originate (Amoroso, 1997).

For these reasons we question the validity of the Hubble mechanism arising from adiabatic expansion, but rather it originates from an inherent spacetime mechanism resulting from the spin exchange spacetime compactification dynamics of quantum gravity.

\section{Photon Propagation and The Vigier Tired Light Hypothesis}

Dissipative redshift mechanisms have remained ad hoc curiosities because of little empirical support and conflict with the apparent strident success of the standard BB model. We expand Vigier's explanation of frequency shift by extending the Sakharov (1968), Puthoff, (1989) vacuum gravitation model to SEQG in a Dirac ether which provides a deeper theoretical framework explaining the origin of non zero rest mass photon anisotrophy in terms of a spin exchange photon propagation process this is a component topology of Planck scale vacuum compactification, i.e. collapse of $\mathrm{N}$ dimensions to the 4-D Minkowski spacetime continuum.

Most physicists today believe the photon is massless because a massive photon would destroy the mathematics of gauge theories and would violate Einstein's theory of special relativity because $M$ would $\rightarrow \infty$ since $\mathrm{u}=\mathrm{c}$. However the

$$
M=M_{0} \frac{1}{\sqrt{1-u^{2} / c^{2}}}
$$

existence of light pressure which has been known for a long time (Nicols \& Hull, 1901) a function of irradiance $I$ over c ( $\mathrm{p}=I / \mathrm{c}$ for absorbed photons and $2 I / \mathrm{c}$ for reflected photons) suggests that photons carry linear momentum and energy which can readily be calculated using Einstein's mass energy relation $h v=m c^{2}$. The de Broglie wavelength relationship for massive particles, taking the accepted value for $\mathrm{R}$ applied to the Vigier mass $m_{\lambda}$ of the photon is:

$$
m_{\lambda}=\frac{n}{\lambda c} \text { taking } \lambda=R \approx 10^{28} \mathrm{~cm} \text { then } m_{\lambda} \approx 2.2 \times 10^{-65} \text { grams }
$$


Where $R$ is the radial size of the universe; and by the uncertainty relation this is the smallest possible photon mass. Further $m \Rightarrow 0$ onlyif $R \Rightarrow \infty$. The de Broglie hypothesis was verified by Davisson \& Germer in 1927 (Fowles, 1989) for the wavelength of a material particle. A photon mass of $10^{-65} \mathrm{~g}$, is in total agreement with Vigier's tired light hypothesis (Vigier, 1990).

It is inherently obvious that the photon is annihilated when brought to rest; therefore it is suggested that the photon has a rest mass with a half life on the order of the Planck time of $10^{-44}$ seconds, which would still preserve gauge in the domain of the standard model of elementary particles and allow for anisotropic vacuum zero point coupling of the photon which if it also occurs in the limit of the Planck time can be a virtual interaction.

\section{The Gravitational Field of a Finite Light Pencil in the Weak-Field Approach}

For the linearized weak-field approximation (WFA) Aichelburg \& Sexl, 1971, assume $m_{\gamma}=0$, point-like and $c=G=1$. For Einstein's field equations: $1 . R_{i k}=1 / 2 g_{i k} R=8 \pi T_{i k}$ and $2 \mathrm{a} . g_{i k} \stackrel{\gamma}{=} \eta_{i k}+2 h_{i k}, 2 \mathrm{~b}$. $\eta_{i k}=\operatorname{diag}(1,-1,-1,-1)$, and 2 c. $\left(h_{i k}\right)^{2} \approx 0$ yield the linearized field equations:

$$
[] \psi^{i k}=8 \pi T^{i k} \quad, \quad \psi^{i k}=-\frac{1}{2} \eta^{i k} h_{l}^{l}
$$

\subsection{DERIVATION OF GRAVITATIONAL FIELD OF RADIATION}

The Vigier mass of the photon is derived utilizing the Tolman, Ehrenfest, Podolsky (TEP) (1931) model of spacetime curvature induced by a finite light pencil. The TEP equations are summarized below; and include Einstein's weak field approximation (WFA) applied to a mass-free radiation field. Accordingly the WPA is linear, deviating only to first order in the Galilean case suggesting that the model is local, i.e., describing spacetime curvature induced by the light pencil in its immediate vicinity. The notation is within the context of classical GR theory.

Only the non-zero components of the energy momentum tensor $T_{\mu}^{v}$ are those in energy density $\rho$. Since the line element integral diverges for an infinitely-long light pencil $\left(L_{p}\right)$ and energy density $\rho$, the pencil length is taken to a finite value $L_{p}$ with $\rho$

also finite. Then the expression for the Galilean deviation yields an elementary function: $h_{\mu}^{\nu}:=\delta^{v \alpha} h_{\mu \alpha}$ with $h:=h_{\alpha}^{\alpha}$ for a $L_{p}$ traveling along the positive axis of an orthogonal Lorentzian 3 - sphere. The linearized WFA from TEP, 1931 is:

$$
\left[h_{\mu}^{v}-\frac{1}{2} \delta_{\mu}^{v} h\right](x, y, z, t)=-4 \iiint \frac{\left[T_{\mu}^{v}\right](\bar{x}, \bar{y}, \bar{z}, t-r)}{r} d \bar{x} d \bar{y} d \bar{z}
$$

Which coupling the metric distribution of matter and energy taken over all elements of spatial volume $d \bar{x} d \bar{y} d \bar{z}$ for time $r$. Using the above WFA solution for the energy momentum tensor $\left[T_{\mu}{ }^{v}\right]$ for electromagnetic radiation for a $L_{p}$ parallel to the x axis, the only density components $\rho$ will be $T_{1}^{1}=-\rho \quad T_{4}^{4}=\rho \quad T_{1}^{4}=-\rho \quad T_{4}^{1}=\rho($ TEP, 1931$)$ 


\subsection{GRAVITATIONAL ACTION OF A LIGHT PENCIL}

The gravitational field in the neighborhood of a finite $L_{p}$ with constant linear energy density $\rho$ passing along the $\mathrm{x}$ axis between a source at $\mathrm{x}=0$ and an absorber at $\mathrm{x}=1$ (TEP, 1931; Wheeler-Feynman, 1945; Cramer, 1986) contributes to the radiation according to:

$$
4 \int \frac{\lfloor\rho\rfloor d V}{r}=-h_{11}=-h_{44}=h_{14}=h_{41}=4 \rho \log \frac{\left\lfloor(l-x)^{\llcorner}+y^{\iota}+z^{\iota}\right\rfloor^{1 / \iota}+l-x}{\left\lceil r^{2}+v,^{2}+r^{2}\right\rceil^{1 / 2}-r}
$$

Equation 2.2 describes the gravitational contribution only in $L_{p}$ neglecting any contribution from the source or absorber ( TEP, 1931) also any internal conditions, vacuum zero point coupling, or other spin exchange which also effect propagation.

Finally for the acceleration of a test particle towards the $L_{p}$ along the negative y direction determined by a geodesic originating midway between the two ends of the pencil, TEP 1931 arrive at the simple result in 4.4. This is significant because the equivalency of the gravitational and inertial mass of a $L_{p}$ justifies the application of the de Broglie relationship in 3.2 to the photon verifying the Vigier hypothesis of $M_{\gamma} \approx 10^{-65} \mathrm{~g}$ !

$$
-\frac{d^{2} y}{d t^{2}}=\frac{2 p l}{v\left[(l / 2)^{2}+v^{2} 7^{1 / 2}\right.}
$$

For which as the de Broglie relationship was applied above in equation (2.2) the Vigier mass $m_{\lambda}$ of $10^{-65} \mathrm{~g}$ is determined. The important characteristic achieved is that conservation of momentum is preserved because as expected the acceleration is exactly twice that calculated from Newtonian theory by taking the equivalence of gravitational and inertial mass!

\section{Internal Structure of the Photon}

According to Einstein rest mass results from external or internal structural motion of a particle. Unlike Fermi materials that are localized in all spacial dimensions and maintain a well developed internal kinetic structure even when at rest, photons immediately release their more open spin structure when brought to rest and immediately dissipate their energy. For photons this internal transformation undergoes oscillation, the rest mass fluctuates harmonically from zero to $>0$ which signifies according to $E=M C^{2}$ a change in energy from inward reflection and interaction with the vacuum to outward displacement through space. Fluctuation in mass-energy is not mysterious as it is generally known that inertial and gravitational masses are an aspect of this movement. At the DESY laboratory recent experimental results have shown that the photon has extra layers of activity (Gribbin, 1995). This is represented in Figure 1.

"In other words, the transformation of "matter" into "energy" is just a change from one form of movement (inwardly, reflecting, toand-fro) into another form (e.g., outward displacement through space. The possibility for objects of zero rest mass exists provided that they are moving at the speed of light. For if rest mass is "inner" movement, taking place even when an object is visibly at rest, it follows that something without "rest mass" has no such inner movement, and that all its movement is outward, in the sense that it is involved in displacement through space. So light does not have the possibility of being "at rest" because it does not poses any such inner movements" (Bohm, 1965). 


\section{Brief Conceptual Introduction to Spin Exchange QG Compactification Dynamics}

Space limitations preclude a brief conceptualization of SEQG, discussed in full in a forthcoming paper (Amoroso, Kafatos, \& Ecimovic, 1998). Photon anisotropy is a requirement of the model. SEQG is based on the fundamental premise that the energetic interplay of mass, inertia, gravitation and spacetime is based on a unified symmetry of internal spin and spin exchange compactification with the photon ultimately being the quantum of action and control. Spin exchange symmetry through the interplay of a unique topological package orders compactification providing a template from which superstring or twistor theory may be completed. One purpose of compactification dynamics is to allow the three sphere of temporal reality to stochastically 'surf' on the superstructure of higher dimensional eternity allowing nonlocal interactions not possible in a Newtonian absolute space or completely described by quantum theory. Stated another way, the domain of quantum uncertainty separates classical linear causality from the nonlinear causality of the unitary field or type III nonlocality.

The localized appearance of compactification has been interpreted as a structure fixed in an early BB era, but SEQG delocalizes compactification in a rich dynamic hyperstructure of continuous spacetime symmetry transformation of constant N Dimensional collapse to the 3-sphere of Minkowski space. The boundary conditions of which determine the speed of light $\mathrm{c}$, the constant acceleration of which balances the GP through the principle of equivalence, and orders the arrow of time. Spacetime is quantized as a discontinuous Planck scale raster determined by the fundamental constants $c, G \& h$. This comprises a basic unit of the Dirac vacuum with the properties of a microcosmic Klein bottle and Planck scale black hole. The Planck constant $h$ is a product of the uncertainty principle; a complement of the Planck length $l_{p l}$ and Planck time $t_{p l}$ comprising the event horizon of nonlocality.

Figure 1. 2D drawing of a $3 D$ view of a $4 D$ hyperstructure. Minkowski spacetime diagram of electric vector only in terms of present moment of 'tiled' Planck units utilizing the WheelerFeynman theory of radiation. The vertices represent absorption \& emission. The observable present is represented by bold lines, and nonlocal components by standard line. Each event is a hyperstructure of Past, Present, and Future interactions, ultimately governed by the quantum of action of SEQG. In the Ref. circle mass and energy fluctuate harmonically during propagation and ZPF coupling.

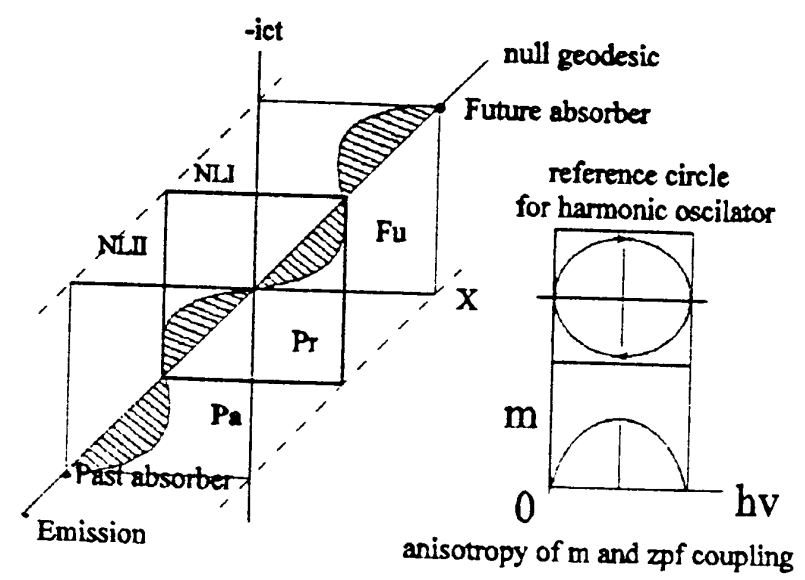

\section{Conclusions}

An anisotropic photon rest mass calculated from both the WFA of classical GR, and the Einstein-de Broglie relationship confirms the Vigier hypothesis. Photon zero point coupling, as required by quantum gravity, has major cosmological implications obviating the big bang by removing the need for an initial singularity in time and still preserves gauge. The GP is equalized by compactification, enabling rigorous calculation of the cosmological constant revealing the arrow of time. Unitarity by its nature must provide pervasive application. Part II in this series of papers applies SEQG to the origin of cosmic microwave background radiation as an intrinsic black body property of cavity quantum electrodynamics inherent in the compactification dynamics of the Dirac ether (Amoroso, 1997b). 


\section{References}

Aichelburg, P.C., Ecker, G. and Sexl, R.U. 1971, Lorentz-covariant Langrangians and causality, Nuovo Cimento B, V. 2B, N.1 p. 63-76.

Amoroso, R.L. 1997a. Noetic field theory: The quantization of mind. Proceedings of 1st International Conference on the Science of Consciousness, Ed. D. Rakovic et al. ECPD, Belgrade.

Amoroso, R.L. 1997b. The origin of cosmic microwave background radiation in the intrinsic fluctuation of vacuum compactification cavity quantum electrodynamics, In: Proceedings of Causality and Locality in Modern Physics, York, Eds: G. Hunter, \& S. Jeffers, 1998 Kluwer.

Amoroso, R.L. \& Kafatos, M. \& Ecimovic, 1998a, Spin exchange quantum gravity as the unitary field, work in progress.

Amoroso, R.L. \& Kafatos, M. \& Ecimovic, 1998b, Quantization of the Dirac vacuum, work in progress.

Bohm, D. 1965. The Special Theory of Relativity, New York, W.A. Benjamin.

Callahan, J.J. 1976. The curvature of space in a finite universe, Scientific American, Aug, 90-99.

Cramer, J.G. 1986. The transactional interpretation of quantum mechanics, Reviews of Modern Physics, Vol 58 No 3; 647-687.

Einstein, A. 1922. Sidelights on Relativity. London, Methuen \& Co.

Gribbin, J. 1995. Schrodinger's Kittens, Boston, Little Brown.

Hoyle, F. \& Narlikar, J.V. 1996. Lectures on Cosmology \& Action at a Distance Electrodynamics, Singapore, World Scientific.

Kafatos, M. 1996, Knowledge limits in cosmology, In: M. Kafatos \& Y. Condo (Eds.) Examining the Big Bang and Diffuse Background Radiations, Netherlands.

Kafatos, M. and Nadeau, R. 1990. The Conscious Universe, New York, Springer-Verlag.

Narlikar, J. V. 1996. The Lighter Side of Gravity, Cambridge, Cambridge Univ. Press.

Narlikar, J.V., Pecker, J.C. \& Vigier, J.P. 1991. Does a possible laboratory observation of a frequency anisotropy of light result from a non-zero photon mass $m$ ? Physics Let A, 154, 5,6,p. 203-9.

Nicols, E.F. and Hull, G.F. 1901. Physical Review, 13, 307.

Puthoff, H.E. 1989, Gravity as a zero-point-fluction force, Physics Review A, 39,2333-2342.

Sakharov, A. 1968, Vacuum quantum fluctuations in curved space and the theory of gravitation, Soviet Physics- Doklady, 12,11: 1040-1041.

Silk, J. 1989, The Big Bang, New York, W.H. Freeman.

Sung, J. Pixels of Space-Time, 1993, Woburn, Scientific Publications.

Tolman, R.C., Ehrenfest, P \& Podolsky, B. 1931, On the gravitational field produced by light, Phys Rev, 37, 602-615.

Vigier, J.P. 1990. Evidence for nonzero mass photons associated with a vacuum-induced dissipative red-shift mechanism. IEEE Trans Plasma Sci, 18,1.p. 64-72.

Wheeler, J.A. \& Feynman, R.P. 1945. Rev. of Modern Physics, 17, 157. 